\title{
Improved Design of Highly Efficient Microsized Lithium-Ion Batteries for Stretchable Electronics
}

Hassan Hafeez ${ }^{1, a}$, Chuanqian Shi ${ }^{2,3, a}$, Chang Min Lee ${ }^{1, a}$, Justin Jesuraj Periyanayagam ${ }^{1}$, Dong Hyun Kim ${ }^{1}$, Myungkwan Song ${ }^{4}$, Chang-Su Kim², Zhanan Zou ${ }^{3}$, Jianliang Xiao ${ }^{3, *}$, Seung Yoon Ryu ${ }^{1,3^{*}}$

${ }^{1}$ Division of Display and Semiconductor Physics, Display Convergence, College of Science and Technology, Korea University Sejong Campus 2511 Sejong-ro, Sejong City, 30019, Republic of Korea

${ }^{2}$ School of Aerospace Engineering and Applied Mechanics, Tongji University, Shanghai, 200092, China

${ }^{3}$ Department of Mechanical Engineering, University of Colorado, 427 UCB, Boulder, CO 80309-0427

${ }^{4}$ Surface Technology Division, Korea Institute of Materials Science (KIMS) Changwon, 51508, Republic of Korea

\begin{abstract}
We introduce a stretchable microsized Li-ion battery with a novel design. The battery fabricated using a facile technique exhibited high performance. A dry-etching process was utilized to pattern lithium phosphorus oxynitride for avoiding the use of a photoresist developer (photolithography), which can damage the amorphous lithium manganese oxide ( $\mathrm{LiMn}_{2} \mathrm{O}_{4}(\mathrm{LMO})$ ) cathode. This allowed us to fabricate the current collectors and electrodes away from the main body of the microsized battery (a parallel structure rather than a stacked, i.e., perpendicular, structure). An enhanced capacity of 150 mAh/g was achieved using the parallel device structure at a charge-discharge average potential of $3.7 \mathrm{~V}$; in comparison, the performance of a device with a stacked structure was significantly inferior. The microsized batteries were utilized to illuminate a white inorganic light-emitting diode with a turn-on voltage of $\sim 2.5 \mathrm{~V}$.

The fabricated devices with the stacked structure were transferred to a mechanically prestrained ( 22.5\%) polydimethylsiloxane polymer via a kinetic transfer process. Upon removal of the prestrain, the shrinkage of the substrate caused buckle formation at the interconnects, and
\end{abstract}


tensile damage to the microsized batteries was circumvented. Simulation (COMSOL) results indicated that most of the strain in the standby form (without applied stretching) was concentrated at the interconnects, preventing significant damage in the main device area. Upon the application of 21\% stretching (load), although the parallel structure exhibits slightly higher maximum strain in the islands, the strain distribution is more effectively managed, indicating the potential of the proposed method for various stretchable and bendable optoelectronic applications.

*Author to whom correspondence should be addressed; e-mail: justie74@korea.ac.kr, ¡ianliang.xiao@colorado.edu

${ }^{a}$ These authors contributed equally to this paper.

Keywords: Lithium-ion microsized rechargeable battery; parallel structure; stacked (perpendicular) structure; stretchable electronics; prestrained PDMS; amorphous $\mathrm{LiMn}_{2} \mathrm{O}_{4}$ (LMO); damage-free fabrication 


\section{Introduction}

Thin-film microsized batteries with Li-based solid electrolytes are experiencing rapid development owing to their significant advantages over batteries with liquid-based electrolytes, e.g., size reduction, elimination of electrolyte-leakage issues, improved battery life, and increased reliability [1, 2]. The desire to implement such batteries in microelectromechanical systems, biomedical, and spacecraft applications has brought considerable challenges regarding the nature of the utilized materials and the design of the device [1, 3]. In this regard, the significant contribution of researchers at Oak Ridge National Laboratory (ORNL) should be acknowledged, as they presented the exceptional benefits of the solid-state electrolyte and demonstrated the use of amorphous lithium phosphorus oxynitride (LiPON) as an electrolyte with vanadium pentoxide $\left(\mathrm{V}_{2} \mathrm{O}_{5}\right)$ as a cathode $[4,5]$. Moreover, the Jet Propulsion Laboratory demonstrated the compatibility of a crystalline $\mathrm{LiCoO}_{2}$ cathode fabricated via radiofrequency (RF) sputtering and annealing at moderate temperatures $\left(<700^{\circ} \mathrm{C}\right)$ with temperature-sensitive substrates [6]. Various other cathode materials have been investigated, such as molybdenum oxide $\left(\mathrm{MoO}_{3}\right)$ [7], fluorinated carbon $\left(\mathrm{CF}_{\mathrm{x}}\right)_{\mathrm{n}}$ [8], and silver vanadium oxide [9]; however, they were found to be unsuitable for highpower applications [10]. Further, Li et al. [11] from ORNL explained that these cathode materials have stability and cycle-life issues; therefore, lithium manganese oxide (LMO)-based electrodes, which have a stable plateau property, and LiPON as a solid electrolyte are preferred owing to their better performances, even though there are many advanced solid electrolytes [3, 10, 11]. However, the aforementioned cathode material $\left(\mathrm{LiMn}_{2} \mathrm{O}_{4}(\mathrm{LMO})\right)$ is sensitive to environmental factors and is prone to damage due to $\mathrm{O}$ components [12] during organic/inorganic chemical treatments or exposure to ultraviolet (UV) light in microfabrication. Various methods have been utilized to efficiently fabricate the devices, including photolithography/etching [13], RF sputtering [14], and 
pulsed laser deposition [3]. However, the search for an improved microsized battery design and a facile fabrication process is still in progress. In particular, with the rapid developments in stretchable and wearable polymer/organic light-emitting diodes (LEDs) [15, 16], biointegrated devices [17], and other electronics [18], considerable attention must be paid to high-performance flexible and stretchable batteries. In this regard, notable contributions have been made recently. For example, Koo et al. [19] demonstrated an all-in-one flexible system incorporating a highperformance bendable Li-ion battery with an LED. Despite the substantial improvements and research efforts in the field of bendable/flexible batteries, significant advancement has not been made in the field of stretchable batteries, owing to the considerable fabrication challenges [20]. Moreover, the stacked device designs have made it difficult to achieve full device capabilities, because of the shorting and oxidation problems for the current collectors [21, 22]. Therefore, significant attention has been paid to redesigning the battery structure for overcoming the stability issues of microsized batteries, e.g., via fabrication of nanowires/nanorods or other porous structures on the electrodes [21, 22]. However, there is an efficiency tradeoff, as the fabrication of such structures reduces the capacity of the microsized batteries.

In this study, we demonstrate a novel design for microsized Li-based batteries that involves relocating the electrodes and current collectors away from the device structure to enhance the device performance. The O-related deterioration of the amorphous LMO cathode can be avoided by replacing the conventional photolithography process with dry-etching for LiPON micropatterning. Fabricated devices with a stacked structure were transferred to a mechanically prestrained ( 22.5\%) polydimethylsiloxane (PDMS) substrate via a kinetic transfer method to evaluate their feasibility for various applications. Upon the removal of the prestrain, the shrinkage of the substrate resulted in buckle formation at the interconnects, preventing damage to the device. 
Analysis of the layer morphology and device performance indicated the potential of the proposed approach in the field of stretchable and wearable electronic devices.

\section{Experiment}

\subsection{Fabrication of stacked structure of microsized battery}

The $\mathrm{Si} / \mathrm{SiO}_{2}$ substrates were first cleaned with a standard clean-1 (SC-1, $\mathrm{NH}_{4} \mathrm{OH}: \mathrm{H}_{2} \mathrm{O}_{2}$ :deionized (DI) water ratio of 1:1:5 at $60{ }^{\circ} \mathrm{C}$ ) solution and a diluted HF (DHF, DI water:HF ratio of 100:1) solution to remove the particle contaminants and native oxides, followed by rinsing with DI water. A thin layer (2 $\mu \mathrm{m})$ of Kapton (polyimide, PI) (Sigma-Aldrich, USA) as a flexible substrate was spin-coated on $\mathrm{Si} / \mathrm{SiO}_{2}$ substrate at $4000 \mathrm{rpm}$ for $60 \mathrm{~s}$, followed by curing at $250{ }^{\circ} \mathrm{C}$ for $0.5-1.5 \mathrm{~h}$. Figure 1 (a) shows a schematic of the fabrication process for the stacked microsized battery. For all the soft-masking processes, a photoresist (PR, AZ 5214, AZ Electronic Materials, Luxembourg) was exposed using conventional photolithography equipment (EVG620, EV Group, Austria) and developed. Then, Pt and Cr (270 nm) current collectors were deposited via sputtering with 100-W power and a 30-sccm Ar flow at 0.67 Pa with deposition rates of approximately 1 and $0.1 \mathrm{~nm} / \mathrm{s}$, respectively. After each deposition step, liftoff was performed; i.e., the PR used as a soft mask was dissolved in acetone and removed. An LMO cathode $(1 \mu \mathrm{m})$ was deposited using RF magnetron sputtering at $100 \mathrm{~W}, 235 \mathrm{~V}$, and 10 Torr, with an $\mathrm{O}_{2}$ :Ar ratio of 22:30. The deposition process was followed by a liftoff process; however, analysis revealed that the deposited LMO cathode was adversely affected by the $\mathrm{O}_{2}$ and organic contaminants utilized in the photolithography. This is discussed in detail in results and discussion part of the manuscript. The solid electrolyte LiPON was sputter-deposited at a rate of $150 \AA / \min (100 \mathrm{~W}, 120 \mathrm{~V}, 4.04$ Torr) 
to a thickness of $450 \mathrm{~nm}$. A Sn anode $(250 \mathrm{~nm})$ and $\mathrm{Cu} / \mathrm{Ti}(250 \mathrm{~nm} / 5 \mathrm{~nm})$ current collectors were deposited using sputter evaporation, under the aforementioned conditions. The encapsulation of the devices was performed with Parylene-C/Ti, where Parylene-C was deposited via thermal evaporation with a base pressure of $\sim 5 \times 10^{-5}$ Torr and a working pressure of $\sim 2 \times 10^{-3}$ Torr. A thin

$(5 \mathrm{~nm})$ layer of Ti was deposited via electron-beam (e-beam) evaporation with a base pressure of $6 \times 10^{-7}$ Torr on top of the Parylene-C layer for enhanced passivation.

\subsection{Fabrication of parallel structure of microsized battery}

To improve the device performance and avoid damage to LMO/LiPON due to the photolithography process, we fabricated a parallel structure, as shown in Fig. 1(b). After the aforementioned LMO deposition process, the LiPON layer was deposited evenly over the whole substrate without masking. The LiPON electrolyte layer was masked with $\mathrm{SiO}_{2}$, and dry-etching of the exposed parts of LiPON was conducted using plasma (5 mT, $20 \mathrm{sccm}, 250 \mathrm{~W}, 5 \mathrm{~min}$ ). In the parallel structure, the $\mathrm{Cu} / \mathrm{Ti}$ current collectors were deposited away from the main device body via the aforementioned sputter evaporation process.

\subsection{Transfer printing of fabricated devices on flexible substrates}

The PDMS mixture was separately prepared by thoroughly mixing a base (Sylgard A) and a curing agent (Sylgard B) and placing the mixture in a $\sim 5.0 \times 10^{-2}$-Torr vacuum to remove bubbles. The liquid PDMS was spin-coated at $1000 \mathrm{rpm}$ for $40 \mathrm{~s}$ onto the fabricated devices, followed by curing at $25^{\circ} \mathrm{C}$ for $24 \mathrm{~h}$. The microsized batteries, along with the PDMS, were quickly peeled off the $\mathrm{Si} / \mathrm{SiO}_{2}$ substrate, and a 50 -nm-thick $\mathrm{SiO}_{2}$ adhesion layer was deposited onto the PDMS via ebeam evaporation with a shadow mask, which was only open in the main device area. A separate 
PDMS substrate $(10 \mu \mathrm{m})$ was prepared and was mechanically prestrained using a custom-made jig, which comprised four clamps with manually adjustable screws. The edges of the PDMS substrate were clamped in the jigs and the substrate was stretched $(22.5 \%$ in both the $\mathrm{X}$ and $\mathrm{Y}$ directions simultaneously). The percentage of stretching was measured according to the difference between the initial and final lengths of the substrate. The fabricated microsized battery devices were finally placed back on the mechanically prestrained flexible PDMS slab via a kinetic transfer process [16]. The release of the prestrain in the PDMS slab resulted in the shrinkage of the device, causing the formation of wavy buckled patterns.

\subsection{Characterization and stretchability simulation analysis}

The surface morphologies of the LMO and LiPON layers, along with their thicknesses, were analyzed using ultrahigh-resolution (UHR) field-emission scanning electron microscopy (FE-SEM, Philips XL30 ESEM and Hitachi S-4700). Additionally, the surface morphologies and amorphous/crystalline nature of the layers were analyzed using transmission electron microscopy (TEM, JEOL-3010) with a selected-area electron diffraction (SAED) tool. The battery performance was analyzed using a Gamry Reference 600 potentiostat (Gamry Instruments, Warminster, PA) in the constant-current discharging mode using a two-electrode configuration, and impedance-frequency measurements were conducted in the three-electrode mode.

The maximum strain distribution on both device structures with and without a biaxial applied load (21\% stretching) was analyzed via finite-element simulation using the commercial software ABAQUS, and the buckling imperfection scale factor for the two microsized batteries was 0.01 . 


\section{Results and discussions}

\subsection{Stacked and parallel microsized battery designs}

As shown in Fig. S1(b), the LMO layer was damaged during the liftoff process for LiPON, probably owing to the aggressive attacking of the PR developer on the LMO layer or the $\mathrm{O}_{2}$ loss due to exposure to UV light [12]. $\mathrm{O}_{2}$-related degradation due to the charge-compensation process was reported to exist during delithiation; however, we speculate that it can occur during microfabrication as well. This damage to the LMO layer was avoided via replacement of the photolithography process by altering the design model for fabrication, as shown in Fig. S2. The photolithography liftoff step for LiPON was replaced by plasma dry-etching of the LiPON layer after complete coverage of the underlying LMO layer. Moreover, the arrangement of the current collectors in the stacked structure has been reported to cause several problems, such as an increase in the internal resistance of the device [21], shorting of the device due to the high contact resistance between the electrode and the current collector [22], and oxidation of cathode due to contact with electrolytes [23]. To avoid such issues, the novel design structure presented in Fig. 1(b) separates the current collectors from the main body of the device. The configurations of the two types of microsized batteries—stacked and parallel—were analyzed using optical microscopy (OM), as shown in Figs. 1(c) and (d), respectively.

\subsection{Analysis of LMO cathode}

The UHR scanning electron microscopy (SEM) images in Fig. 2(a) and (b) show the thickness, uniformity, and smooth deposition of each layer, with well-defined layer boundaries. Among all the utilized layers, LMO cathodes tend to form crystals when annealed at high temperatures. Initial approaches suggested that the crystalline LMO was preferable in thin-film batteries owing to its 
excellent plateau. However, the crystalline form of LMO was analyzed to present problems, such as capacity fading and cycling issues due to the phase change of crystalline LMO to irreversible spinel-like structures [24-26]. Therefore, to enhance the lifetime and performance of the microsized battery, the amorphous nature of LMO is preferable [24-26]. X-ray diffraction (XRD) analysis (Fig. 2(c)) of LMO did not indicate a sharp peak; instead, a broad peak around $2 \theta=20^{\circ}$ was detected. This broad peak suggests the amorphous nature of the LMO [27] and is attributed to the minor presence of the tetragonal spinel structure of LMO (JCPDS card No. 89-0118). Similarly, the surface morphology (SEM image shown in the inset of Fig. 2(c)) of the LMO layer indicates a smooth surface (absence of crystal formation) with little roughness variation. For further analysis, the composition of the LMO cathode was determined using X-ray photoelectron spectroscopy (XPS) (Fig. 2(d)). The spectrum exhibited Li (1s/6), O (1s/4), and Mn (2p/3) peaks around 280, 520 , and $630 \mathrm{eV}$, respectively, in agreement with previous reports [27, 28]. No other peaks were detected (except for a small peak corresponding to $\mathrm{C}$ at $284 \mathrm{eV}$ ), indicating the high purity of the LMO layer [27, 28]. According to the XPS analysis, the exact composition of the elements in the LMO cathode was $\mathrm{Li}_{2.13} \mathrm{Mn}_{1.15} \mathrm{O}_{4}$, even though the SEM and XRD analyses indicated the amorphous nature of the LMO layer. Diffused rings, along with bright spots, were observed in the SAED pattern (Fig. 2(e)). The bright spots were observed in a continuous cycle rather than being symmetrical, indicating the minor presence of the amorphous spinel structure, as previously mentioned. The TEM analysis (Fig. 2(f)) also indicated the slight roughness of the surface of the LMO layer, corroborating the previous results for the morphology of the layer. Overall, the results suggest that the LMO layer had an amorphous form with a low crystallinity, and is in agreement with the XRD and SEM analyses.

\subsection{Analysis of LiPON solid electrolyte}


The microcrystalline or amorphous nature of the LiPON is of utmost importance because the induction of crystallinity in LiPON has been observed to reduce the ionic conductivity and degrade the electrochemical properties of the thin film [29, 30]. In contrast to the case of LMO, the SAED analysis for LiPON (Fig. 3(a)) did not reveal any spots or diffused rings, indicating that the LiPON had a completely amorphous form. This is in accordance with the smooth LiPON layer observed via TEM (Fig. 3(b)). The quantitative analysis of LiPON via XPS (Fig. 3(e)) revealed the representative peaks of $\mathrm{Li}(1 \mathrm{~s} / 9), \mathrm{P}(2 \mathrm{p} / 8), \mathrm{N}(1 \mathrm{~s} / 6)$, and $\mathrm{O}(1 \mathrm{~s} / 5)$ at 120, 280, 398, and $520 \mathrm{eV}$, respectively [30, 31]. The bonding environment for O could have included P-O-P, Li-O-P, and P=O bonds [30], and the N 1s peak appears to contain three Gaussian-Lorentzian components, which is in agreement with previous observations [30]. The composition was analyzed to be $\mathrm{Li}_{2.13} \mathrm{PO}_{3.24} \mathrm{~N}_{0.81}$, indicating a high-quality LiPON layer without impurities. The SEM surfacemorphology analysis (Fig. 3(d)) indicated that the top layer (Sn anode) was dense, with uniform grain growth of the irregular structure, which is beneficial for microsized battery applications. Figures 3(e) and (f) show the results of impedance analysis using alternating-current impedance spectroscopy. The LiPON film was deployed in a sandwich structure between $\mathrm{Pt} / \mathrm{Cr}$ and Pt layers, with $\mathrm{Si} / \mathrm{SiO}_{2}$ as the substrate (inset Fig. 3(e)). In this device structure, the impedance could be categorized in two frequency regions. The resistance to the charge transfer and capacitance present at the LiPON/electrode interface resulted in the response at low frequencies $\left(<10^{3}\right)$ [32]. However, at higher frequency $\left(>10^{3}\right)$ the semi-circle was obtained, which indicated that the impedance values in that region were related to the resistance and capacitance of the amorphous LiPON layer itself [32]. Hence, the ionic conductivity of this amorphous LiPON film was calculated using the following equation by fitting the Nyquist plot: 


$$
\sigma=d /(R \cdot A),
$$

where, $d$ is the LiPON film thickness, which was measured to be $450 \mathrm{~nm}$ (Fig. 2(a)); $\mathrm{R}$ is the LiPON film resistance, which was taken as the value of $\mathrm{Z}$ at the frequency where - $\mathrm{Z}$ ' reached a local minimum; and A is the area of metal in contact. The ionic conductivity of LiPON was calculated to be $1.4 \times 10^{-6} \mathrm{~S} / \mathrm{cm}$, which is close to the value reported by ORNL researchers $(3 \times$ $10^{-6} \mathrm{~S} / \mathrm{cm}$ ) [2]. This indicates that a high-quality and high-purity LiPON layer was deposited through our process; thus, high performance of the thin-film microsized battery was expected.

\subsection{Device performance comparison of microsized batteries}

A performance comparison between the microsized batteries with the stacked and parallel structures is shown in Figs. 4(a) and (b), respectively. The charge-discharge behavior of the stacked microsized battery exhibited a plateau at $\sim 3.3 \mathrm{~V}$, indicating that the $\mathrm{Li}^{+}$ions were well extracted by the oxidation of the LMO cathode. The Li ions migrated toward the anode, where they were reduced, resulting in the formation of the Li film. For efficient battery operation, a flat discharge curve is ideal; however, a significant decrease in the discharge curve was observed, indicating a very low capacity of $10 \mathrm{mAh} / \mathrm{g}$. As the discharge cycle concluded with the oxidation of the Li film, reducing the LMO cathode back to the initial state [1], the discharge failure is attributed to the inability of the LMO cathode to undergo the reduction process. As previously mentioned, the damage to the LMO cathode caused by O contaminants [12] could be one of the reasons for its deterioration. This damage occurred during the photolithography process and was due to the utilization of organic-inorganic liquids. This over-oxidation might have contributed to the charge cycle; however, in the discharge cycle, it hindered the reduction process and caused the 
failure of the microsized battery. To avoid such contamination of the cathode and the shorting of the device [21, 22], we redesigned the microsized battery with a parallel structure. We excluded the photolithography process during the deposition of LiPON and fabricated current collectors away from the main device body. The charge-discharge curves for the parallel structure (Fig. 4(b)) showed exceptional improvement. The charge cycle started at approximately $3.7 \mathrm{~V}$ and exhibited a linear curve, indicating the efficient oxidation of the LMO cathode and the subsequent formation of the Li film at the anode. Interestingly, the discharge cycle was also successful; the discharge curve exhibited a slow decrease caused by the gradual oxidation of the anode and the successful reduction of the cathode. Consequently, a high capacity of $\sim 150 \mathrm{mAh} / \mathrm{g}$ was achieved with the parallel structure of the microsized battery, which is comparable to the previously reported device efficiencies. The parallel-structured microsized battery was employed to turn on a white LED, and Fig. 4(c) shows photographs of the parallel microsized battery connected to an LED (before and after turn-on). The LED device turned on at a low voltage of $2.5 \mathrm{~V}$ with a current of only 10-20 $\mu \mathrm{A}$, indicating the capability of the microsized battery to operate at a low discharge rate, as shown in Fig. 4(d). The stability of the parallel microsized battery was tested under numerous chargedischarge cycles at approximately $13 \mu \mathrm{A} / \mathrm{cm}^{2}$ (Figs. 4(e) and (f)). Even though there is a minor discrepancy in the measurement as after first discharge cycle, the capacity of the battery was a bit increased $(<10 \mathrm{mAh} / \mathrm{g})$. However, the minor difference is negligible and the overall trend of the final charge-discharge cycle well matched that of the initial cycle, indicating a high battery stability. It should be noted that due to smaller contact areas of the electrodes, probes were used to obtain the electrical measurements, however, crocodile connectors were used for the illumination of the LED. The change in the connector types might somewhat alter the overall connection resistance and the performance of the microsized battery under load might be slightly different. 
Moreover, we demonstrated the potential of the proposed microsized batteries for stretchable, wearable electronics. Figure S3 shows the steps involved in the kinetic transfer printing process $[16,33]$ of the stacked (as a representative) stretchable microsized batteries. In this study, only stacked stretchable microsized batteries were examined, because the well-defined symmetrical main device area (square, not rectangular) could only be fabricated with the stacked structure via the symmetrical shadow masking process for the deposition of the $\mathrm{SiO}_{2}$ adhesion layer. The parallel structure has a nonsymmetrical main device area, separated from the current collector. However, in the stacked structure, the device performance with regard to stretchable electronics was significantly degraded owing to the adverse effects of the chemicals involved in the photolithography process on the LMO. If a stretchable microsized battery device with a parallel structure was fabricated with a well-defined symmetrical bridge and main device area, better performance could be achieved. After fabrication of the thin-film microsized battery array on a $\mathrm{Si} / \mathrm{SiO}_{2}$ substrate, a slab of PDMS (1-3 mm thick) was independently prepared, which served as an elastomeric stamp [16, 33]. The cured, sticky PDMS slab was brought into conformal contact with the device and was quickly peeled off, removing and detaching the whole device from the bottom $\mathrm{Si} / \mathrm{SiO}_{2}$ substrate. A 50 -nm-thick $\mathrm{SiO}_{2}$ adhesion layer was deposited on the main device area only, via e-beam evaporation and shadow masking [34]. The quick peel-off was due to the weak adhesion of the PI to the $\mathrm{Si} / \mathrm{SiO}_{2}$ substrates. The peeled-off stamp, along with device and $\mathrm{SiO}_{2}$ adhesion layer, was then slowly placed on mechanically prestrained PDMS (22.5\% strain) to ensure proper adhesion. Upon the removal of the mechanical strain (the substrate was unclamped from the jigs), the compressive stresses generated in the substrate resulted in the formation of buckles at the interconnects, allowing the device to be stretched to various dimensions. The OM images of the fabricated stretchable microsized battery (stacked, Fig. S4) indicate that the 
microsized battery device was successfully transferred to the mechanically prestrained PDMS and that the interconnects were flat (straightened out). The main battery structure deposited on $\mathrm{SiO}_{2}$, which encountered the PDMS substrate and interconnects, was formed by the shadow mask and avoided contact with the PDMS. This type of fabrication method minimizes the strain effect on the main device body and compensates the strain at the buckling, causing bending. This bending is observed at the interconnects in the magnified OM image (Fig. S4(b)), along with the absence of cracks/defects (Fig. S4(c)) caused by the kinetic transfer or the removal of the prestrain from the PDMS substrate. Additionally, Fig. S4(d) shows the thin-film microsized battery array on the PDMS substrate, indicating the absence of wrinkles/stretches caused by the application or removal of the prestrain.

\subsection{Finite-element simulations for evaluating mechanical performance}

To investigate the mechanical performance, we performed finite-element analysis (FEA) on both device configurations in the buckled (standby, without stretching) and biaxially stretched (flat, 21\% stretching) states using the commercial software package ABAQUS. The results shown in Fig. 5. The PDMS substrate was modeled as an isotropic material using three-dimensional stress elements (C3D8), and the microsized batteries (Fig. S5-S7) with meshed island-interconnect structures were modeled using composite shell elements (S4). To simulate the post-buckling behavior of the interconnects, buckling imperfections were introduced into the interconnects, with the buckling modes determined via buckling analysis. As shown in Figs. 5(a) and (b), the strains are low compared with the failure strains of the constitutive materials. The strains at the interconnects of both devices are far higher than those in the islands because the islands are significantly stiffer than the soft substrate to which they are bonded, while the interconnects are 
free to deform in the out-of-plane direction, and their effective stiffness is far lower than that of the islands. When the prestrain is released, the stiff islands restrain the deformation in the substrate, while the interconnects buckle and form arc shapes, consuming most of the deformation in the substrate. This deformation mechanism prevents the functional but brittle islands from breaking and provides mechanical stretchability to the device as a whole. The maximum strain at the interconnects of the parallel structure (2.2\%) is slightly higher than that for the stacked structure (2.1\%). This is due to the slightly wider spacing between the islands in the stacked structure, which yields less interconnect deformation in the out-of-plane direction during buckling.

The fracture strains of copper and platinum are $\varepsilon_{f(C u)}=45 \%$ [35] and $\varepsilon_{f(P t)}=4 \%$ [36], respectively, and the maximum principal strains of metal bridges are lower than their fracture strains, thus the structural design of the battery meets the operational requirements. The fatigue properties could be calculated through Coffin-Manson relationship [37],

$$
\frac{\Delta \varepsilon}{2}=\varepsilon_{f}\left(2 N_{f}\right)^{c}+\frac{\sigma_{f}}{E}\left(2 N_{f}\right)^{b},
$$

where the Young's moduli and fracture stresses of copper and platinum are: $E_{C u}=130000 \mathrm{MPa}$, $\sigma_{f(C u)}=220 \mathrm{MPa}, E_{P t}=168000 \mathrm{MPa}$, and $\sigma_{f(P t)}=1800 \mathrm{MPa}$, and the coefficients are $b=-0.12$ and $c=-0.6$. In this study, the strain magnitudes for two different designs are $\Delta \varepsilon s t a c k e d=0.021$ and $\Delta \varepsilon_{\text {parallel }}=0.022$. According to Eq. (2), the number of loading cycles before material failure is obtained as 295 for copper and 25 for platinum in the stacked structure, and 271 for copper and 21 for platinum in the parallel structure. However, in most application conditions, the device doesn't go through the full strain magnitude. If the strain magnitude is reduced to $50 \%$ of the maximum strains in copper and platinum, the number of loading cycles before material failure increases to 1025 for copper and 530 for platinum in the stacked structure, and 941 for copper and 408 for platinum in the parallel structure. 
The right frames of Figs. 5(a) and (b) show the strain contours in the islands due to the buckling of the interconnects. Although the parallel structure exhibits a higher maximum strain in the islands, the strain distribution is more effectively managed, with the maximum strain confined outside the main device area (indicated by the red dotted lines) owing to the design of the device. Figures 5(c) and (d) show the strain distributions in the stacked and parallel structures when they are subject to $21 \%$ biaxial stretching, which causes the buckled interconnects to be nearly fully flattened. The results indicate that the maximum strains in the islands and interconnects of both the stacked and parallel structures are considerably reduced; thus, the mechanical designs of both structures are suitable for stretchable devices.

To guide the design of multilayer interconnects for optimized mechanical performance, the neutral mechanical plane, where the strain due to bending is zero, of the interconnect cross section is calculated. As shown in Fig. S7, the interconnect is composed of three layers: PI, metal (Cu or Pt), and PDMS, whose Young's moduli and thicknesses are as follows: $E_{P I}=1.72 \mathrm{MPa}, h_{1}=2 \mu \mathrm{m}$, $E_{\text {metal }}\left(E_{C u}=130000 \mathrm{MPa}\right.$ and $\left.E_{P t}=168000 \mathrm{MPa}\right), h_{2}=0.25 \mu \mathrm{m}, E_{P D M S}=1.8 \mathrm{MPa}$, and $h_{3}=10$ $\mu \mathrm{m}$. The position of the neutral mechanical plane of the trilayer is obtained by assuming plane stress condition as

$$
h=\frac{E_{P D M S} h_{3}\left(2 h_{1}+2 h_{2}+h_{3}\right)+E_{\text {metal }} h_{2}\left(2 h_{1}+h_{2}\right)+E_{P I} h_{1}^{2}}{2\left(E_{P D M S} h_{3}+E_{\text {metal }} h_{2}+E_{P I} h_{1}\right)} .
$$

The interconnect should be designed such that the neutral mechanical plane is at the middle plane of the metal layer to minimize the strain in this layer and to optimize the mechanical performance. For PI/Cu/PDMS and PI/Pt/PDMS trilayers, the positions of the neutral mechanical planes are 
calculated to be both at $h=2.127 \mu \mathrm{m}$, which is very close to the location of the middle plane of the metal layers in experiment $h^{\prime}=2.125 \mu \mathrm{m}$. It's worth pointing out that a recently develop traction-free model could be adopted to provide improved prediction of mechanical properties of laminated ribbon structures [38]. Therefore, the design of the interconnects is reasonable and effectively reduces the stress and deformation of the metal films. The novel parallel design for microsized batteries proposed in this work has good potential for stretchable, wearable, and biomedical applications owing to its superior electrical and mechanical properties.

\section{Conclusions}

A facile and novel fabrication process for stretchable microsized Li-ion batteries was introduced, which allows minimization of the defects caused by the O contaminants in the LMO cathode via replacement of the photolithography process with dry-etching of LiPON. Additionally, the shorting of the devices due to the contact resistance between the current collector and the electrodes/electrolytes (stacked structure) was avoided by moving the current collectors away from the main device (parallel structure). Consequently, the discharge capacity of the microsized battery was significantly improved, from $10 \mathrm{mAh} / \mathrm{g}$ (stacked structure) to $150 \mathrm{mAh} / \mathrm{g}$ (parallel structure). Moreover, the battery was successfully employed to power a white LED at a low operation voltage (2.5 V), and charge-discharge cycles revealed the improved efficiency and stability of the parallelstructured microsized battery. The fabricated devices with a stacked structure were transferred to a mechanically prestrained PDMS substrate, which induced the buckling of the interconnects for stretchability. Finite-element simulations indicated that the maximum strains in both structures were on the buckled interconnects upon the release of the prestrain, which prevented the mechanical failure of the functional but brittle device areas (islands). The simulation results for 
both structures subjected to $21 \%$ biaxial stretching indicated significantly reduced strain values for the parallel device structure, indicating that the proposed design can be efficiently employed for various wearable and stretchable electronic applications.

\section{Authors information}

\section{Corresponding Author}

*Prof. Dr. Seung Yoon Ryu

${ }^{1}$ Division of Display and Semiconductor Physics, Display Convergence, College of Science and Technology, Korea University Sejong Campus, 2511 Sejong-ro, Sejong City, 30019 Republic of Korea, ${ }^{3}$ Department of Mechanical Engineering, University of Colorado, 427 UCB, Boulder, CO 80309-0427, Tel: +82-44-860-1375, justie74@korea.ac.kr

*Prof. Dr. Jianliang Xiao

${ }^{3}$ Department of Mechanical Engineering, University of Colorado, 427 UCB, Boulder, CO 803090427, jianliang.xiao@colorado.edu

\section{Author Contributions}

${ }^{a}$ These authors contributed equally to this paper.

Competing financial interests (Notes):

The authors declare no competing financial interests.

\section{Acknowledgements}


This research was supported by Basic Science Research Program through the National Research Foundation of Korea (NRF) funded by the Ministry of Education (NRF-2014R1A6A1030732 and 2017R1A2B4005583). This work was also supported by "Human Resources Program in Energy Technology” of the Korea Institute of Energy Technology Evaluation and Planning (KETEP), granted financial resource from the Ministry of Trade, Industry \& Energy, Republic of Korea (No. 20184030201910).

\section{ORCID Ids}

Seung Yoon Ryu: https://orcid.org/0000-0003-3914-1145

Jianliang Xiao: https://orcid.org/0000-0001-5378-0096

Hassan Hafeez: https://orcid.org/0000-0001-9647-008X 


\section{Figure Captions}

Figure 1. (a) Schematic of the stacked structure on the $\mathrm{Si} / \mathrm{SiO}_{2}$ substrate. (b) Schematic of the parallel microsized battery with the redesigned structure. (c) OM image of the stacked structure showing the LiPON, LMO cathode, and Pt/Cr current collectors. (d) OM image of the parallel structure showing the $\mathrm{Cu} / \mathrm{Ti}$ current collectors located away from the main device body.

Figure 2. Characterization of the LMO cathode. (a) UHR FE-SEM cross-sectional image of the stacked device, indicating the thickness of each layer. (b) UHR FE-SEM cross-sectional image at a lower magnification, indicating the uniformity of the deposited films. (c) XRD analysis of LMO, with the inset showing a UHR FE-SEM image of the deposited layer. (d) Quantitative XPS analysis results. (e) SAED results, indicating minor spinel LMO and a major amorphous layer. (f) TEM image of the deposited LMO cathode.

Figure 3. Characterization of the LiPON solid electrolyte. (a) SAED analysis indicating the completely amorphous nature of LiPON. (b) TEM analysis. (c) XPS analysis indicating the exact elemental composition of LiPON. (d) UHR FE-SEM image of the Sn anode, showing the porosity and density of the layer. (e) Impedance measurement for LiPON, with the sandwich device structure shown in the inset. (f) Ionic-conductivity measurement/calculations for LiPON.

Figure 4. Performance of the microsized battery device. (a) Charge-discharge cycles for the stacked structure. (b) Charge-discharge cycles for the parallel structure. (c) Photographs of the white LED operated using the parallel microsized battery. (d) Current-voltage analysis for the turn-on of the white LED using the parallel microsized battery. (e) Stability analysis of the parallel structure. (f) Stability trend of the 
parallel structure under charge-discharge cycling.

Figure 5. Strain distributions in the stacked and parallel structures obtained via the FEA simulation for the buckled forms of the (a) stacked structure and (b) parallel structure and under $21 \%$ biaxial stretching for the (c) stacked structure and (d) parallel structure. 


\section{References}

[1] West W, Whitacre J, White V and Ratnakumar B 2001 Fabrication and testing of all solidstate microscale lithium batteries for microspacecraft applications Journal of Micromechanics and Microengineering 1258

[2] Bates J, Dudney N, Neudecker B, Ueda A and Evans C 2000 Thin-film lithium and lithiumion batteries Solid State Ionics 135 33-45

[3] Albrecht D, Wulfmeier H and Fritze H 2016 Preparation and Characterization of cLiMn2O4 Thin Films prepared by Pulsed Laser Deposition for Lithium-Ion Batteries Energy Technology 4 1558-64

[4] Bates J, Gruzalski G and Luck C 1993 Rechargeable solid state lithium microbatteries. In: Micro Electro Mechanical Systems, 1993, MEMS'93, Proceedings An Investigation of Micro Structures, Sensors, Actuators, Machines and Systems. IEEE.: IEEE) pp 82-6

[5] Bates J, Gruzalski G, Dudney N and Luck C 1992 New amorphous thin-film lithium electrolyte and rechargeable microbattery. In: Power Sources Symposium, 1992., IEEE 35th International: IEEE) pp 337-9

[6] West W, Whitacre J, Brandon E and Ratnakumar B 2001 Lithium micro-battery development at the Jet Propulsion Laboratory IEEE Aerospace and Electronic Systems Magazine 16 31-3

[7] Julien C, Nazri G, Guesdon J, Gorenstein A, Khelfa A and Hussain O 1994 Influence of the growth conditions on electrochemical features of $\mathrm{MoO} 3$ film-cathodes in lithium microbatteries Solid State Ionics 73 319-26

[8] Watanabe N and Fukuda M 1970 Primary cell for electric batteries. Google Patents)

[9] Takeuchi E S and Piliero P 1987 Lithium/silver vanadium oxide batteries with various silver to vanadium ratios Journal of power sources 21 133-41

[10] Wang Y, Liu B, Li Q, Cartmell S, Ferrara S, Deng Z D and Xiao J 2015 Lithium and lithium ion batteries for applications in microelectronic devices: A review Journal of Power Sources 286 330-45

[11] Li J, Ma C, Chi M, Liang C and Dudney N J 2015 Solid electrolyte: the key for highvoltage lithium batteries Advanced Energy Materials 51401408

[12] Cho E, Kim K, Jung C, Seo S-W, Min K, Lee H S, Park G-S and Shin J 2017 Overview of the Oxygen Behavior in the Degradation of Li2MnO3 Cathode Material The Journal of Physical Chemistry C 121 21118-27

[13] Oukassi S, Salot R and Pereira-Ramos J-P 2007 Microfabricated Solid State Thin Film Lithium Batteries. In: MATERIALS RESEARCH SOCIETY SYMPOSIUM PROCEEDINGS: Warrendale, Pa.; Materials Research Society; 1999) p 24

[14] Hwang B-J, Wang C-Y, Cheng M-Y and Santhanam R 2009 Structure, morphology, and electrochemical investigation of LiMn2O4 thin film cathodes deposited by Radio frequency sputtering for lithium microbatteries The Journal of Physical Chemistry C $\mathbf{1 1 3}$ 11373-80

[15] White M S, Kaltenbrunner M, Głowacki E D, Gutnichenko K, Kettlgruber G, Graz I, Aazou S, Ulbricht C, Egbe D A and Miron M C 2013 Ultrathin, highly flexible and stretchable PLEDs Nature Photonics 7811

[16] Hafeez H, Zou Z, Kim D H, Shin J Y, Song M, Kim C-S, Choi W J, Song J, Xiao J and Ryu S Y 2017 Multiaxial wavy top-emission organic light-emitting diodes on thermally 
prestrained elastomeric substrates Organic Electronics 48 314-22

[17] Kim D-H, Ghaffari R, Lu N and Rogers J A 2012 Flexible and stretchable electronics for biointegrated devices Annual review of biomedical engineering 14 113-28

[18] Fan J A, Yeo W-H, Su Y, Hattori Y, Lee W, Jung S-Y, Zhang Y, Liu Z, Cheng H and Falgout L 2014 Fractal design concepts for stretchable electronics Nature communications 53266

[19] Koo M, Park K-I, Lee S H, Suh M, Jeon D Y, Choi J W, Kang K and Lee K J 2012 Bendable inorganic thin-film battery for fully flexible electronic systems Nano letters 12 4810-6

[20] Liu W, Song M S, Kong B and Cui Y 2017 Flexible and stretchable energy storage: recent advances and future perspectives Advanced materials $\mathbf{2 9}$

[21] Min H-S, Park B Y, Taherabadi L, Wang C, Yeh Y, Zaouk R, Madou M J and Dunn B 2008 Fabrication and properties of a carbon/polypyrrole three-dimensional microbattery Journal of Power Sources 178 795-800

[22] Gowda S R, Leela Mohana Reddy A, Zhan X, Jafry H R and Ajayan P M 2012 3D nanoporous nanowire current collectors for thin film microbatteries Nano letters 12 1198202

[23] Oltean G, Nyholm L and Edström K 2011 Galvanostatic electrodeposition of aluminium nano-rods for Li-ion three-dimensional micro-battery current collectors Electrochimica Acta 56 3203-8

[24] Iriyama Y, Yada C, Abe T, Ogumi Z and Kikuchi K 2006 A new kind of all-solid-state thinfilm-type lithium-ion battery developed by applying a DC high voltage Electrochemistry communications 8 1287-91

[25] John X J, Hui Y, Gaurav J and Jingsi Y 2004 Amorphous manganese oxide remains amorphous upon lithium intercalation and cycling Electrochemistry communications 6 892-7

[26] Xu J J, Kinser A J, Owens B B and Smyrl W H 1998 Amorphous manganese dioxide: a high capacity lithium intercalation host Electrochemical and solid-state letters 1 1-3

[27] Ming H, Yan Y, Ming J, Adkins J, Li X, Zhou Q and Zheng J 2014 Gradient V2O5 surfacecoated LiMn2O4 cathode towards enhanced performance in Li-ion battery applications Electrochimica Acta 120 390-7

[28] Chen S, Chen Z and Cao C 2016 Mesoporous spinel LiMn2O4 cathode material by a softtemplating route Electrochimica Acta 199 51-8

[29] Liu W-Y, Fu Z-W, Li C-L and Qin Q-Z 2004 Lithium phosphorus oxynitride thin film fabricated by a nitrogen plasma-assisted deposition of E-beam reaction evaporation Electrochemical and solid-state letters 7 J36-J40

[30] Pichonat T, Lethien C, Tiercelin N, Godey S, Pichonat E, Roussel P, Colmont M and Rolland P A 2010 Further studies on the lithium phosphorus oxynitride solid electrolyte Materials Chemistry and Physics 123 231-5

[31] Nimisha C, Rao K Y, Venkatesh G, Rao G M and Munichandraiah N 2011 Sputter deposited LiPON thin films from powder target as electrolyte for thin film battery applications Thin Solid Films 519 3401-6

[32] Jiménez A R, Nölle R, Wagner R, Hüsker J, Kolek M, Schmuch R, Winter M and Placke T 2018 A step towards understanding the beneficial influence of a LIPON-based artificial SEI on silicon thin film anodes in lithium-ion batteries Nanoscale 10 2128-37

[33] Meitl M A, Zhu Z-T, Kumar V, Lee K J, Feng X, Huang Y Y, Adesida I, Nuzzo R G and Rogers J A 2006 Transfer printing by kinetic control of adhesion to an elastomeric stamp Nature materials 533 
[34] Khang D-Y, Jiang H, Huang Y and Rogers J A 2006 A stretchable form of single-crystal silicon for high-performance electronics on rubber substrates Science 311 208-12

[35] Ring T A, Feeney P, Boldridge D, Kasthurirangan J, Li S and Dirksen J A 2007 Brittle and ductile fracture mechanics analysis of surface damage caused during CMP Journal of The Electrochemical Society 154 H239-H48

[36] Tang X, Jonnalagadda K, Chasiotis I, Lambros J, Polcawich R, Pulskamp J and Dubey M 2007 Effect of Strain-rate on the Mechanical Behavior of Pt-Films for MEMS. In: 2007 SEM Annual Conference \& Exposition on Experimental and Applied Mechanics,

[37] Merchant H, Minor M and Liu Y 1999 Mechanical fatigue of thin copper foil Journal of Electronic Materials 28 998-1007

[38] Yin S and Su Y 2019 A traction-free model for the tensile stiffness and bending stiffness of laminated ribbons of flexible electronics Journal of Applied Mechanics $\mathbf{8 6} 051011$ 
Figure 1.

(a)

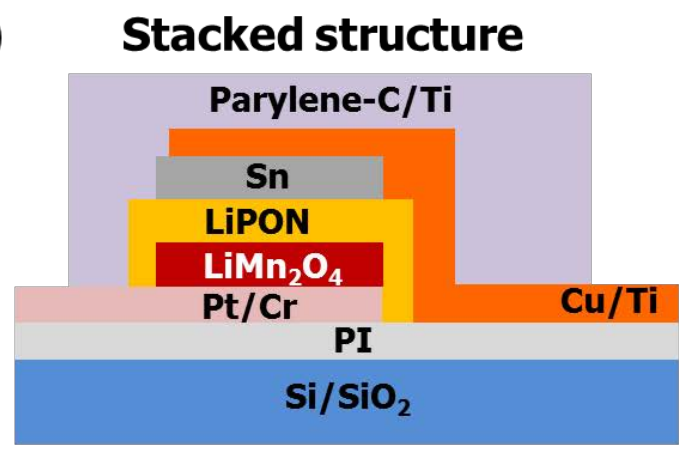

(c)

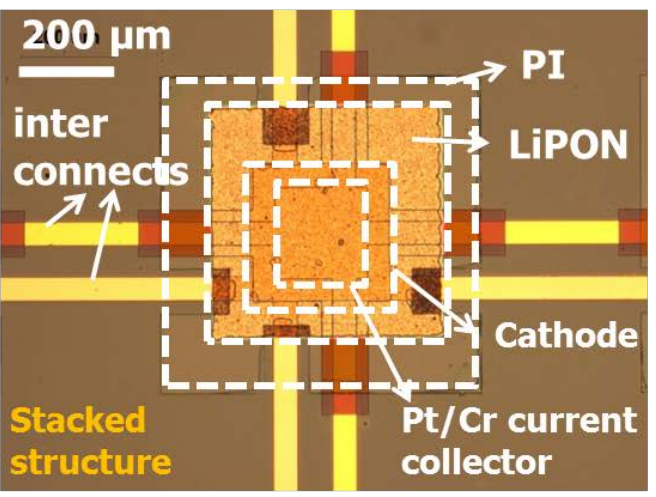

(b)

\section{Parallel structure}

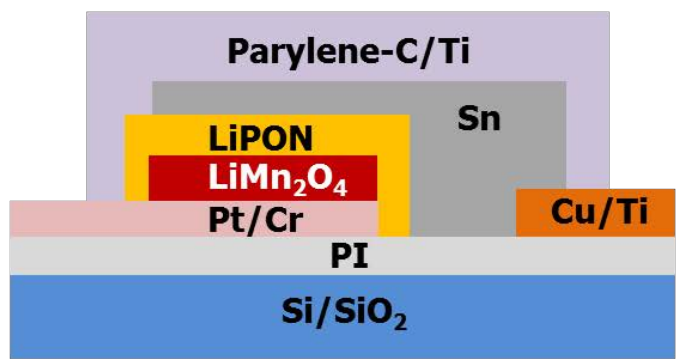

(d)

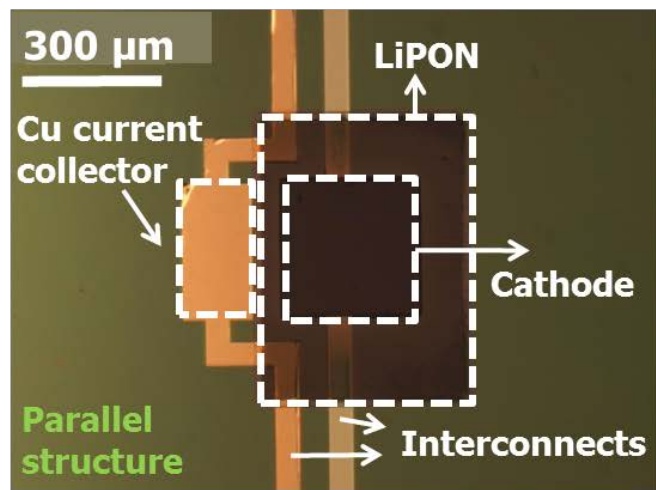


Figure 2.

(a)
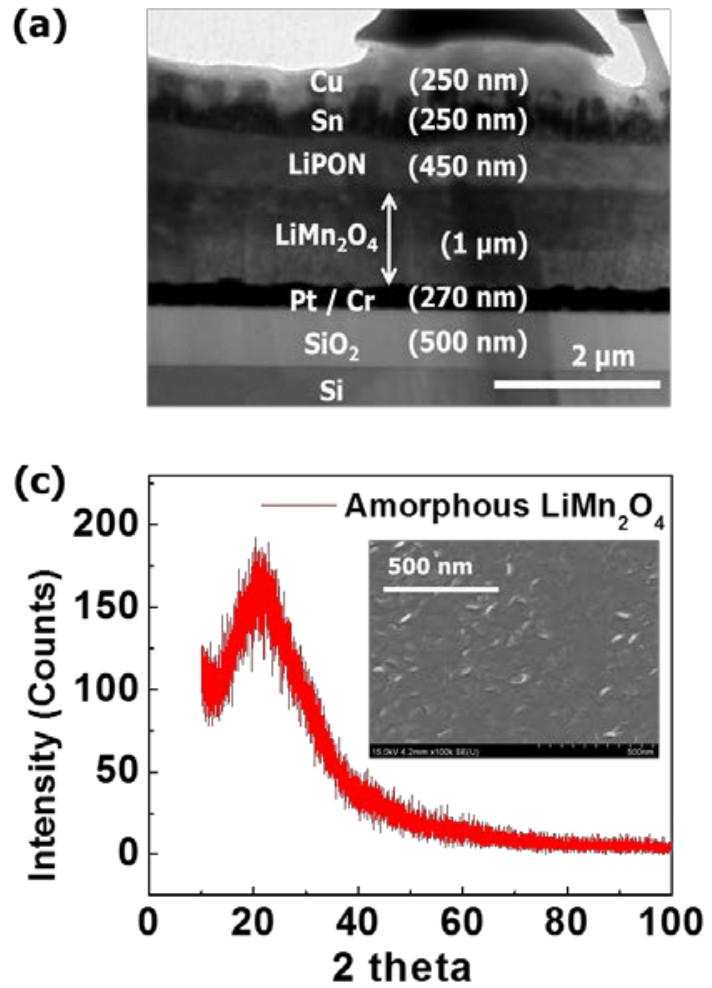

(e)

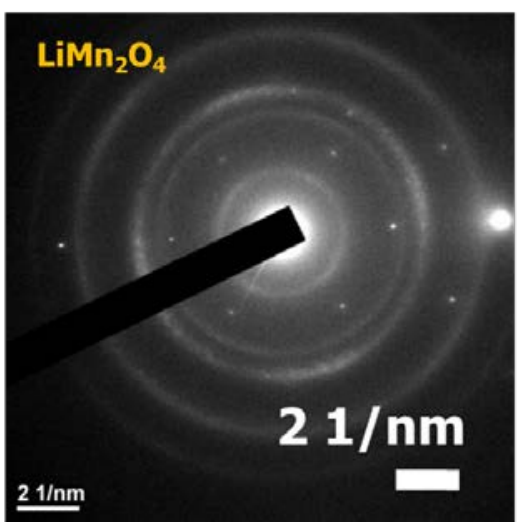

(b)
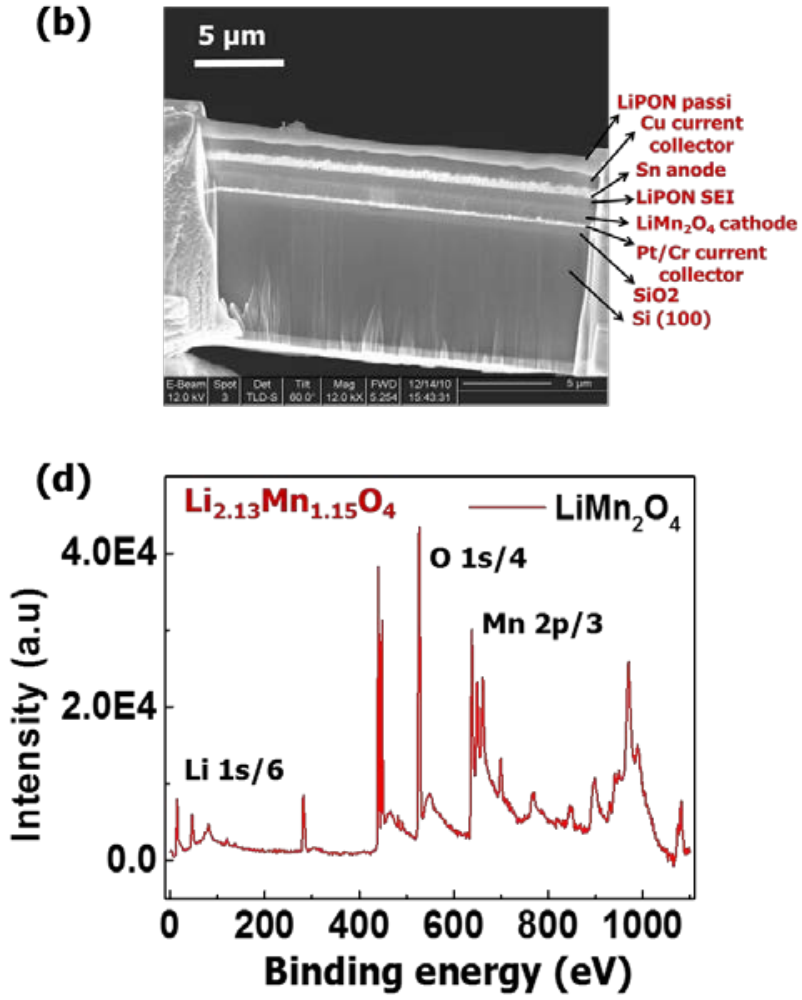

(f)

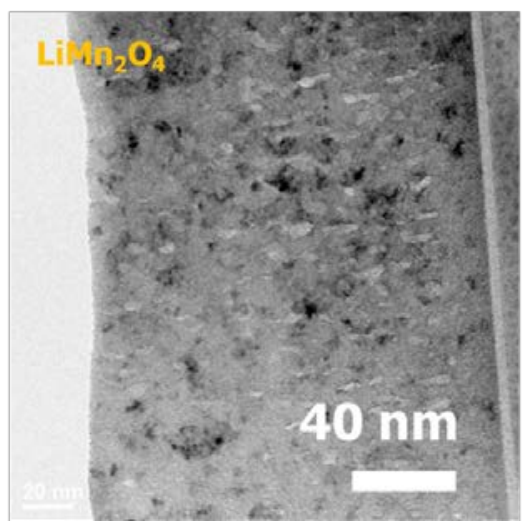


Figure 3.

(a)

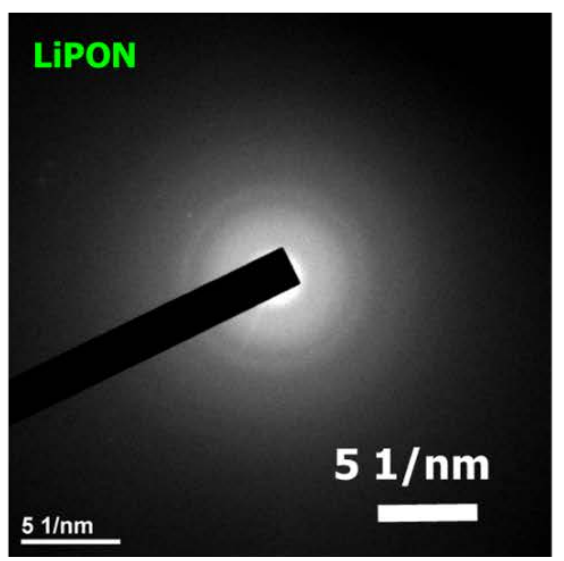

(c)
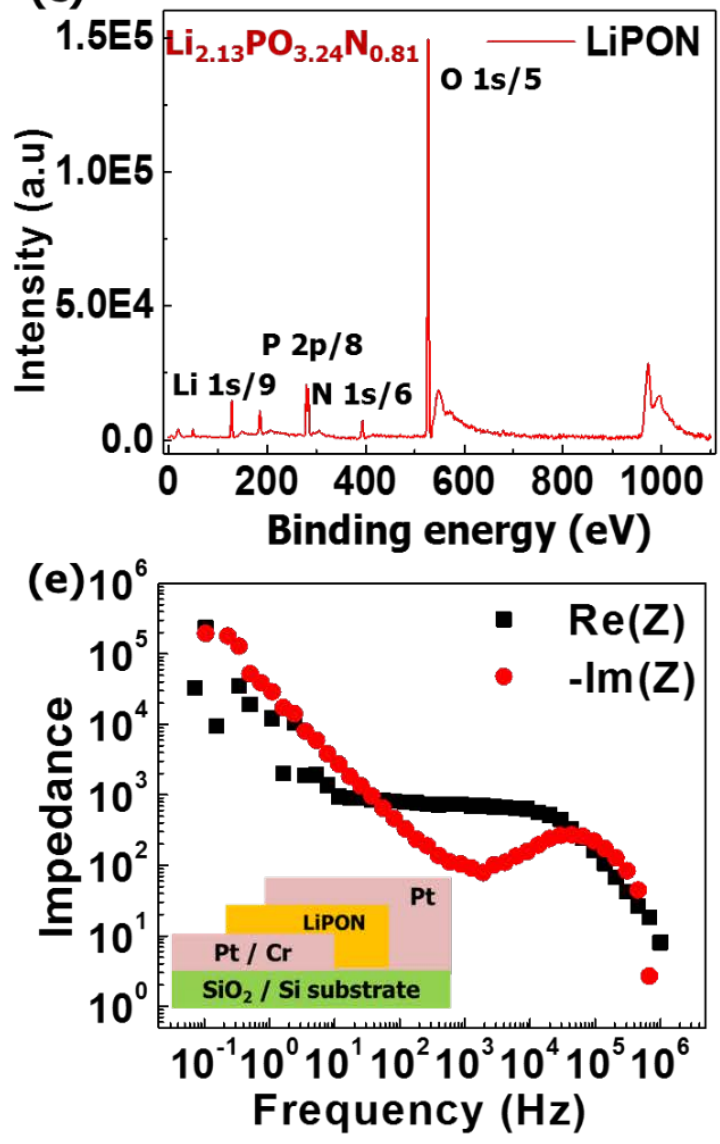

(b)

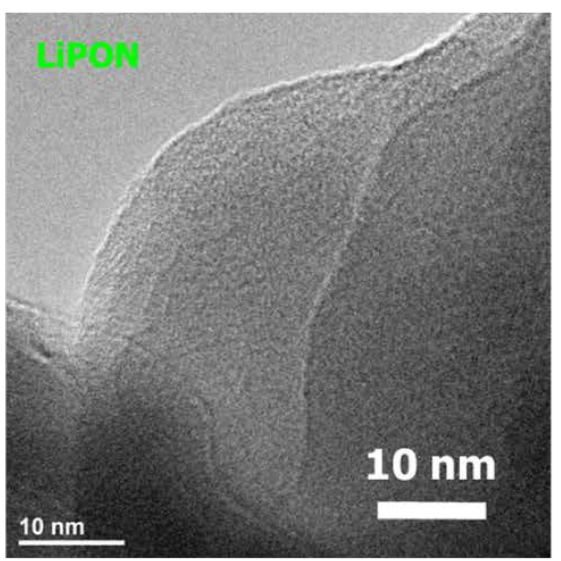

(d)
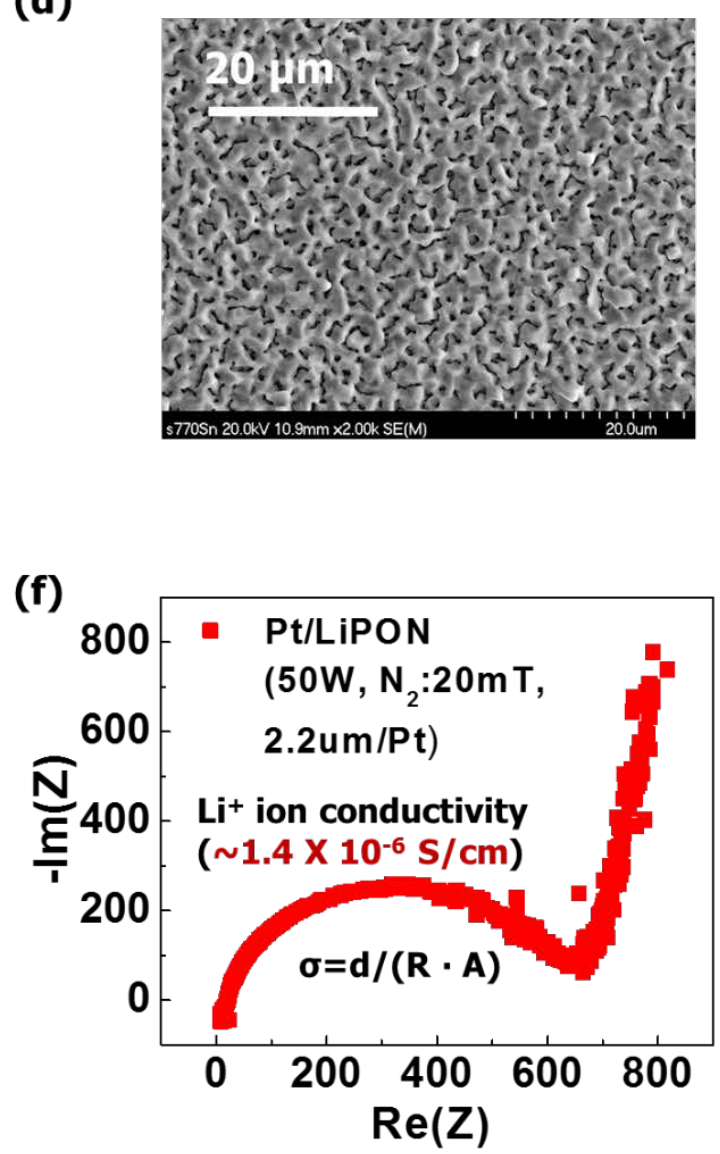
Figure 4.
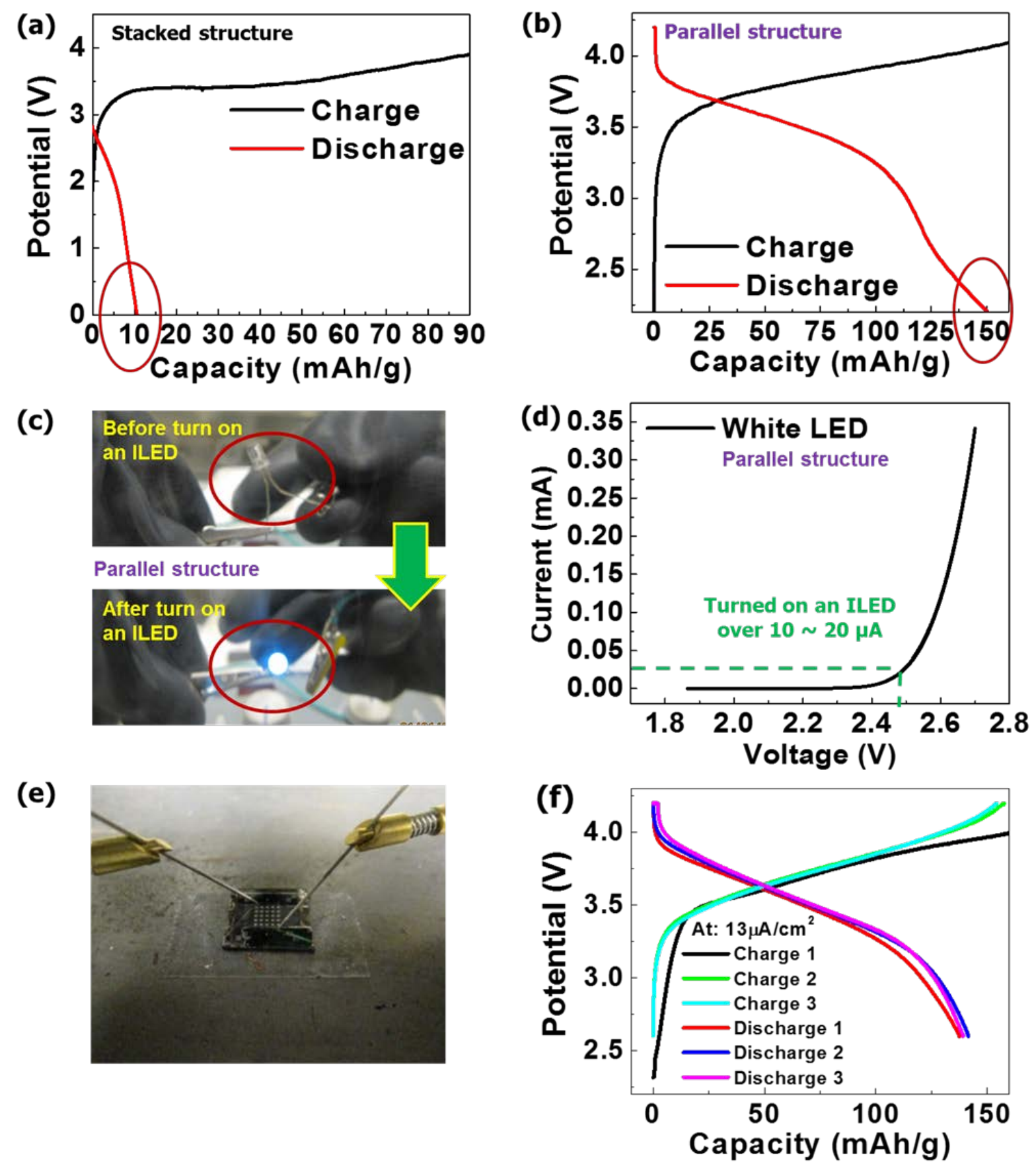
Figure 5.

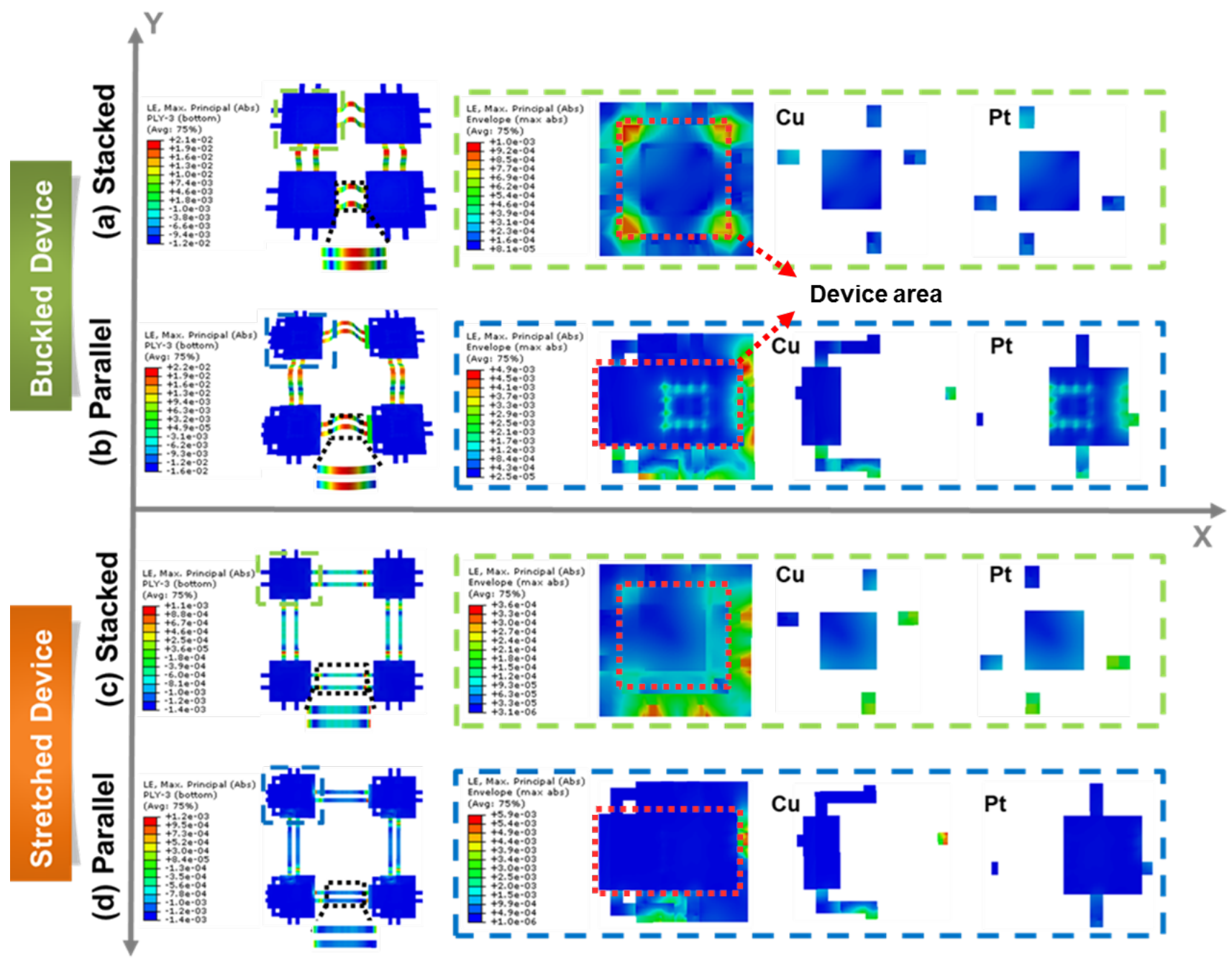




\title{
Supporting Information
}

\section{Improved Design of Highly Efficient Microsized Lithium-Ion Batteries for Stretchable Electronics}

\author{
Hassan Hafeez ${ }^{1, a}$, Chuanqian Shi',3,a, Chang Min Lee ${ }^{1, a}$, Justin Jesuraj Periyanayagam¹, Dong Hyun Kim \\ Myungkwan Song ${ }^{4}$, Chang-Su Kim4, Zhanan Zou ${ }^{3}$, Jianliang Xiao ${ }^{3, *}$, Seung Yoon Ryu ${ }^{1,3^{*}}$ \\ ${ }^{1}$ Division of Display and Semiconductor Physics, Display Convergence, College of Science and Technology, Korea \\ University Sejong Campus 2511 Sejong-ro, Sejong City, 30019, Republic of Korea \\ ${ }^{2}$ School of Aerospace Engineering and Applied Mechanics, Tongji University, Shanghai, 200092, China \\ ${ }^{3}$ Department of Mechanical Engineering, University of Colorado, 427 UCB, Boulder, CO 80309-0427 \\ ${ }^{4}$ Surface Technology Division, Korea Institute of Materials Science (KIMS) Changwon, 51508, Republic of Korea
}


Figure S1. Schematic of the fabrication of the stacked device structure. (a) Liftoff process for Pt and LMO. (b) Liftoff process for LiPON, showing the possibility of damage to the LMO cathode due to oxygen components from organic chemicals during photolithography. (c) LiPON deposition step. (d) Sn anode patterning on LiPON using a shadow mask. (e) Liftoff process for fabricating $\mathrm{Cu}$ current collectors. (f) Final stacked microsized battery device structure (PI/Pt/Cr/LMO/LiPON/Sn/Cu).

(a) Liftoff process for Pt \& $\mathrm{LiMn}_{2} \mathrm{O}_{4}$

$\mathrm{LiMn}_{2} \mathrm{O}_{4}$

$\mathrm{Pt} / \mathrm{Cr}$

PI

(b) Liftoff process for LiPON $\left(\mathrm{O}_{2}\right.$ components damaged $\left.\mathrm{LiMn}_{2} \mathrm{O}_{4}\right)$

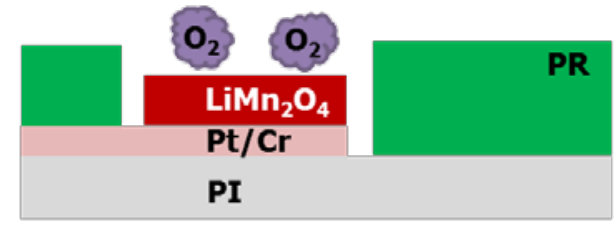

(c)

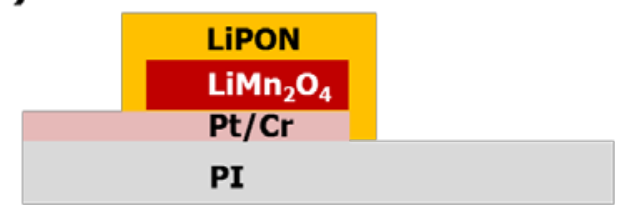

(d) Sn patterning using: shadow mask

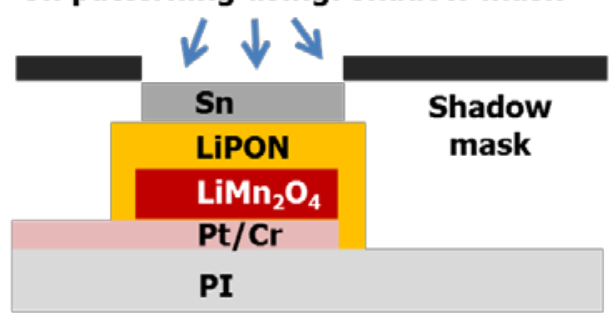

(e) Liftoff process for Cu current collector

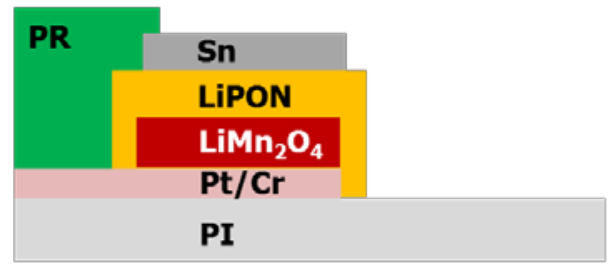

(f)

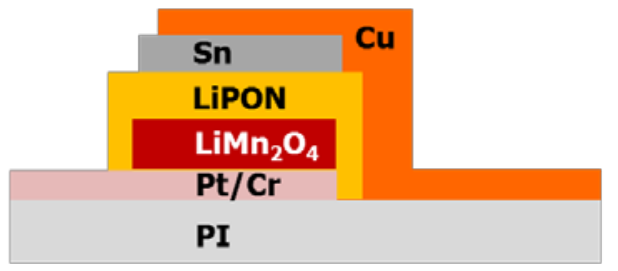

The schematic of the fabrication process for the stacked structure shows that during the liftoff process for LiPON, acetone was used to remove the PR from the (LMO) layer, as the presence of organic/ $\mathrm{O}_{2}$ components could damage the LMO layer. This may have induced poor contact with the overlaying LiPON layer or adversely affected the electronic properties of the LMO layer. Additionally, if the current collectors are placed in the stacked structure, the high contact resistance and oxidation of the cathode degrade the device performance. 
Figure S2. Schematic of the fabrication of the parallel device structure. (a) Liftoff process for Pt, $\mathrm{Cu}$, and $\mathrm{LMO}$. The $\mathrm{Cu} / \mathrm{Ti}$ layers were deposited in the initial steps, in contrast to the case of the stacked structure, for moving the $\mathrm{Cu} / \mathrm{Ti}$ current collector away from the main body of microsized battery device. (b) LiPON layer deposition over the whole substrate and dry-etching of LiPON instead of photolithography. (c) Schematic showing the full removal of the residual layer. (d) Sn patterning using a shadow mask, which was the final step of the parallel microsized battery fabrication.

(a)

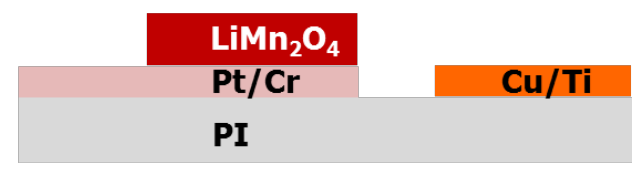

(b)

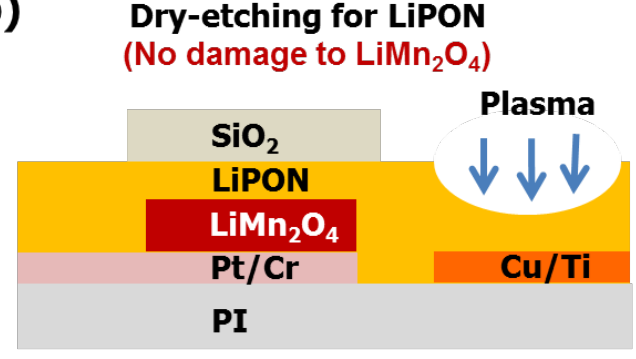

(c) Complete removal of residual layer

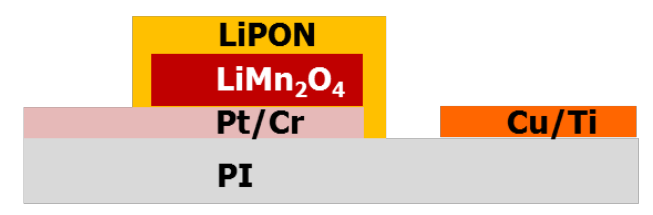

(d) Sn patterning using: shadow mask

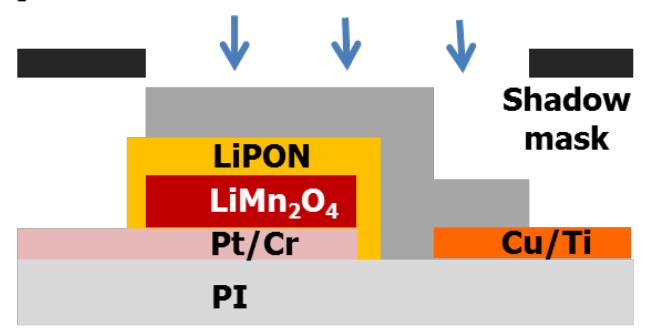

To avoid damage to the LMO layer (Fig. S1) during the fabrication process, first, the liftoff process was replaced by plasma dry-etching of the LiPON layer. The $\mathrm{SiO}_{2}$ masking layer for the dryetching of LiPON was fabricated via the liftoff process. Second, the current collectors were fabricated away from the main device structure, improving the quality of the LMO layer and the overall device performance by eliminating shorting effects caused by interconnects. 
Figure S3. Schematic of the kinetic transfer printing method for the microsized battery (stacked structure). (a) Fabrication of the microsized battery on $\mathrm{Si} / \mathrm{SiO}_{2}$. (b) Quick peel-off of the device with a PDMS stamp. (c) Slow peel-back on mechanically prestrained (substrate stretched using clamps) PDMS (22.5\%). (d) Final devices on the stretchable and flexible substrate with and without prestrain.

(a) Microbattery Fabrication

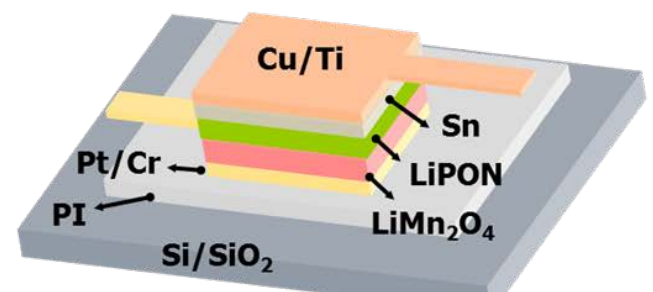

(c) Slow Peel-back

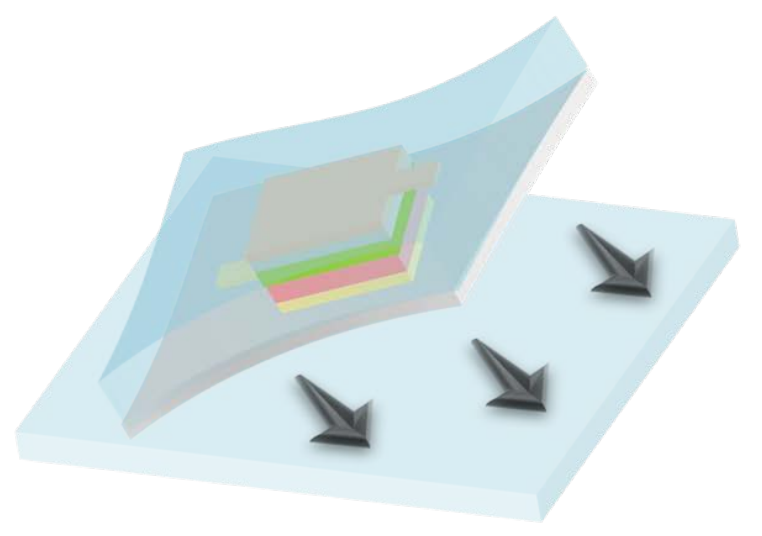

Pre-strained PDMS $22.48 \%$ (b) Quick peel-off

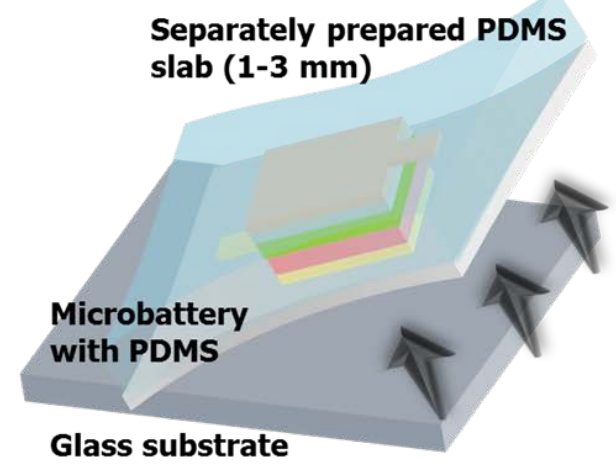

(d) With and without prestrain
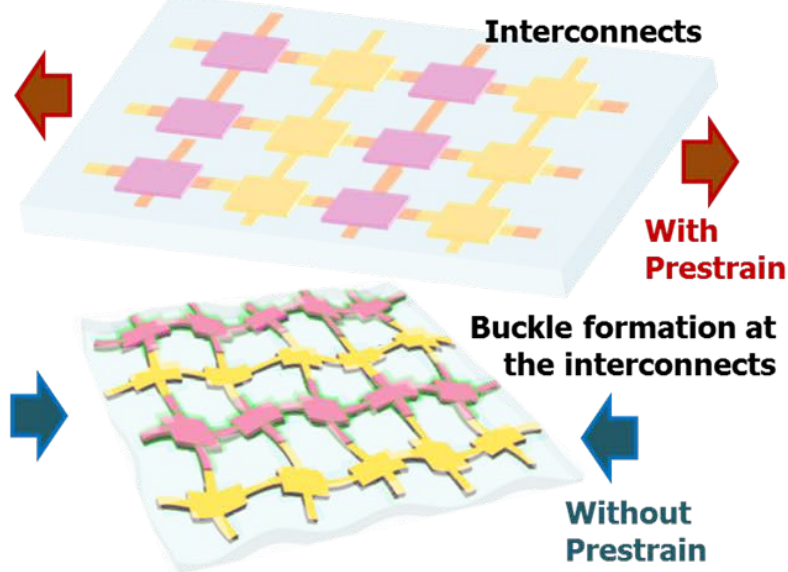

The thin-film battery devices were fabricated on a $\mathrm{Si} / \mathrm{SiO}_{2}$ substrate and a separately prepared PDMS slab (1-3 mm thick). After curing, the PDMS slab was brought into contact with the fabricated device and was quickly peeled off along with the device and the $\mathrm{SiO}_{2}$ layer at the bottom, which was deposited as an adhesion layer using a shadow mask. The flexible PDMS slab provided 
not only a method for stripping off the device but also mechanical stability. The microsized battery was peeled back on a mechanically prestrained ( 22.48\%) PDMS substrate using the kinetic transfer printing method, as the device was sandwiched between two PDMS layers. Upon the release of the prestrain, the shrinkage in the bottom PDMS layer resulted in the formation of buckles at the interconnects, and the microsized battery structures retained their flatness. 
Figure S4. Stacked stretchable battery array. (a-c) OM images of the stacked microsized battery at different magnifications, indicating the defect-free fabrication of the devices using the kinetic transfer printing method. The interconnects exhibited buckle formation. (d) Photograph of the stacked microsized battery array on the elastomeric substrate.

(a)

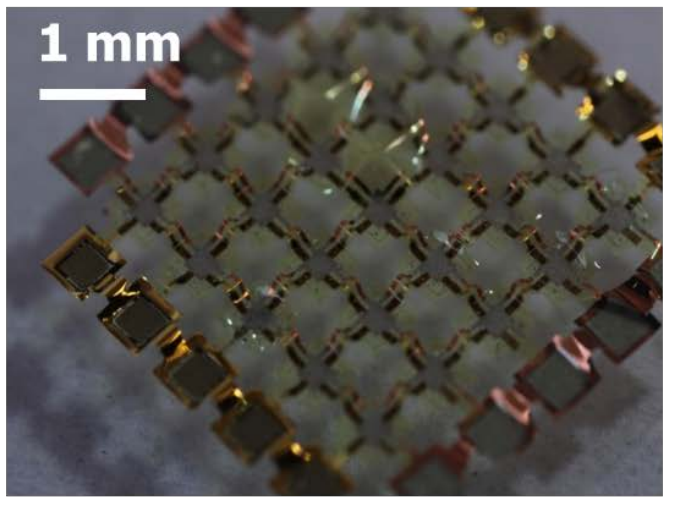

\section{(c)}

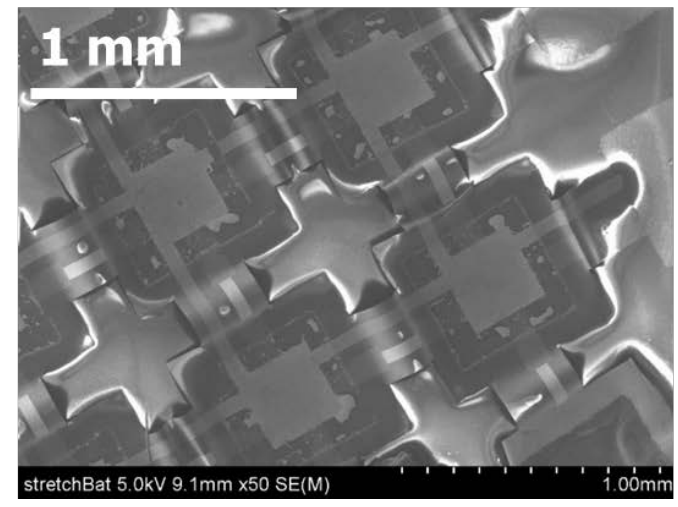

(b)

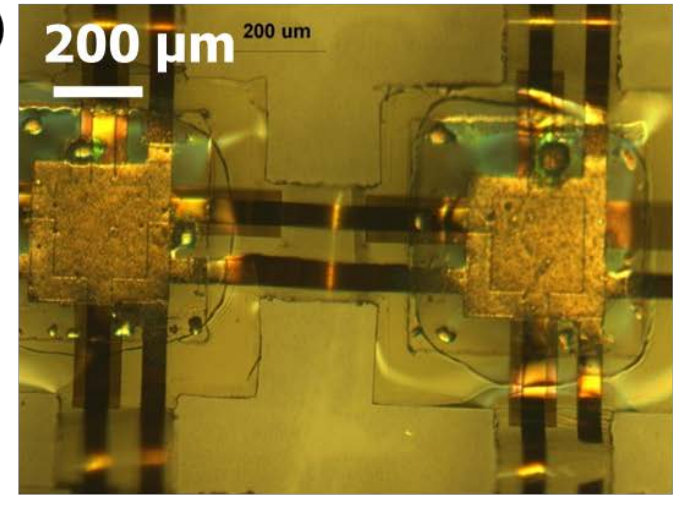

(d)

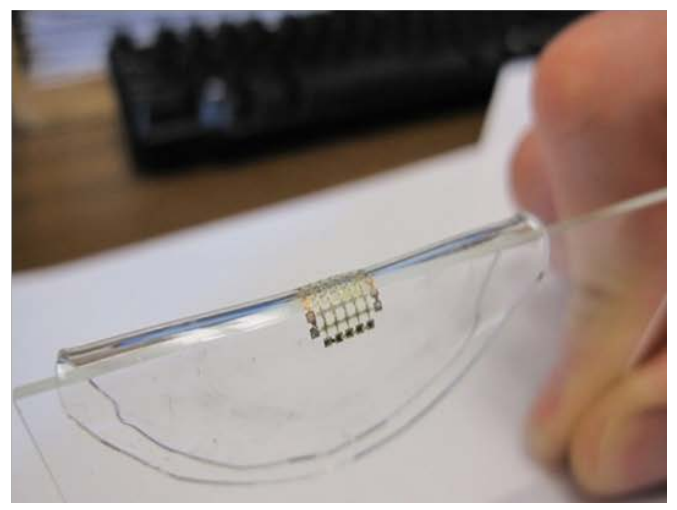

As shown in the OM images, the fabricated microsized battery array had no visible cracks or defects, owing to the incorporation of the device between PDMS layers. The formation of buckles at the interconnects is observed in Fig. S4(b), where no apparent strain or deformation is detected. The photograph in Fig. S4(d) shows the flexibility of the fabricated devices, indicating that the kinetic transfer printing method is efficient for fabricating stretchable/flexible microsized batteries with high stability. 
Figure S5. Device designs (top view) for the stacked and parallel battery structures for demonstrating the buckle formation.

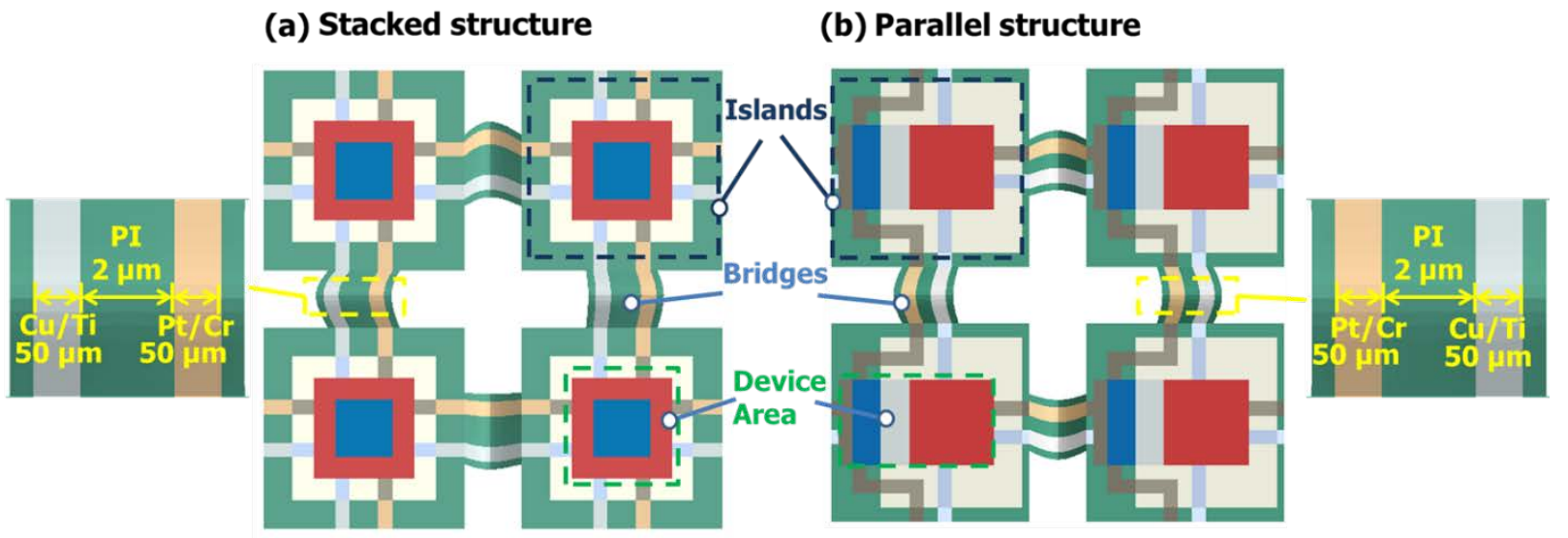

The top view of the devices shows the islands (device areas) and buckled interconnects. The interconnects were designed as sandwiched structures with $\mathrm{PDMS} / \mathrm{Cu} / \mathrm{Ti} / \mathrm{PI}$ and $\mathrm{PDMS} / \mathrm{Pt} / \mathrm{Cr} / \mathrm{PI}$. The width of the $\mathrm{Cu} / \mathrm{Ti}$ and $\mathrm{Pt} / \mathrm{Cr}$ interconnects was $~ 50 \mu \mathrm{m}$, and the distance between them was $\sim 100 \mu \mathrm{m}$ in the stacked structure and $\sim 50 \mu \mathrm{m}$ in the parallel structure. The thicknesses of the PDMS, Cu/Ti, Pt/Cr, and PI interconnects were $10 \mu \mathrm{m}, 250 \mathrm{~nm}, 270$ nm, and $2 \mu \mathrm{m}$, respectively. 
Figure S6. Schematics of the devices on the PDMS substrate, illustrating the stretching mechanism used for the FEA simulations.

(a) Unloading $22.48 \%$ pre-strain

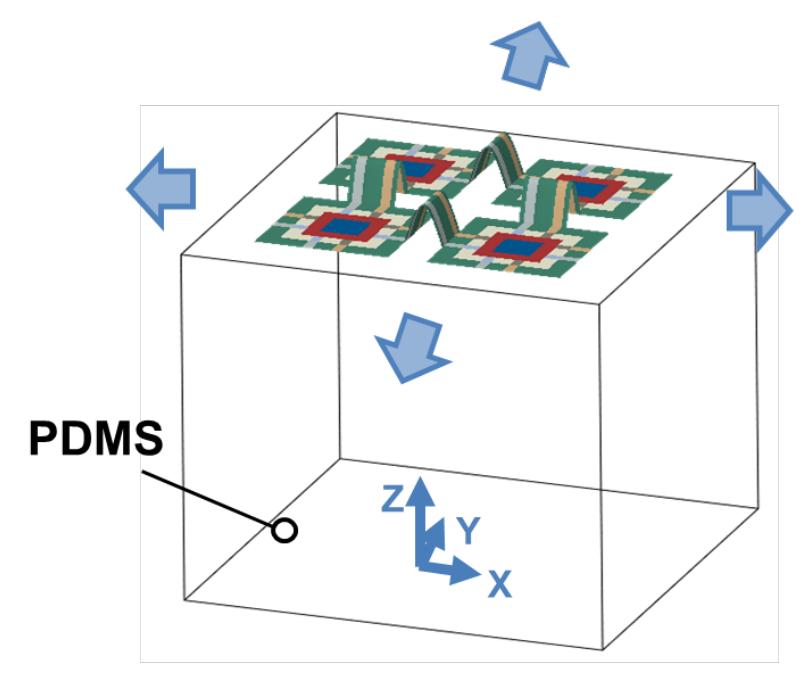

(b) After unloading, stretch device to $21 \%$ strain

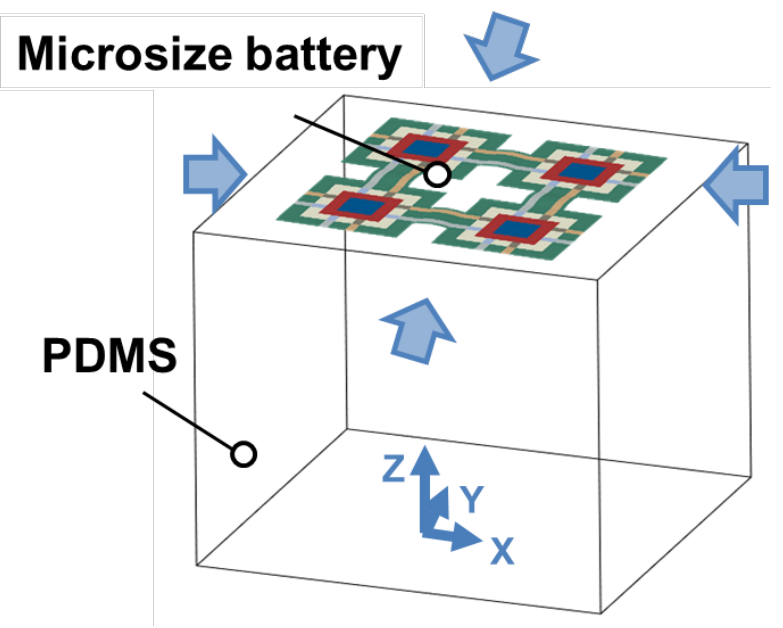

A side view of the devices on the PDMS substrate is shown in Fig. S6. The PDMS layer is $2 \mathrm{~mm}$ thick. Figure S6(a) shows the buckles formed when the prestrain was released after the kinetic printing transfer process. Figure S6(b) shows the device after 21\% biaxial stretching. 
Figure S7. Schematic of the interconnect design.

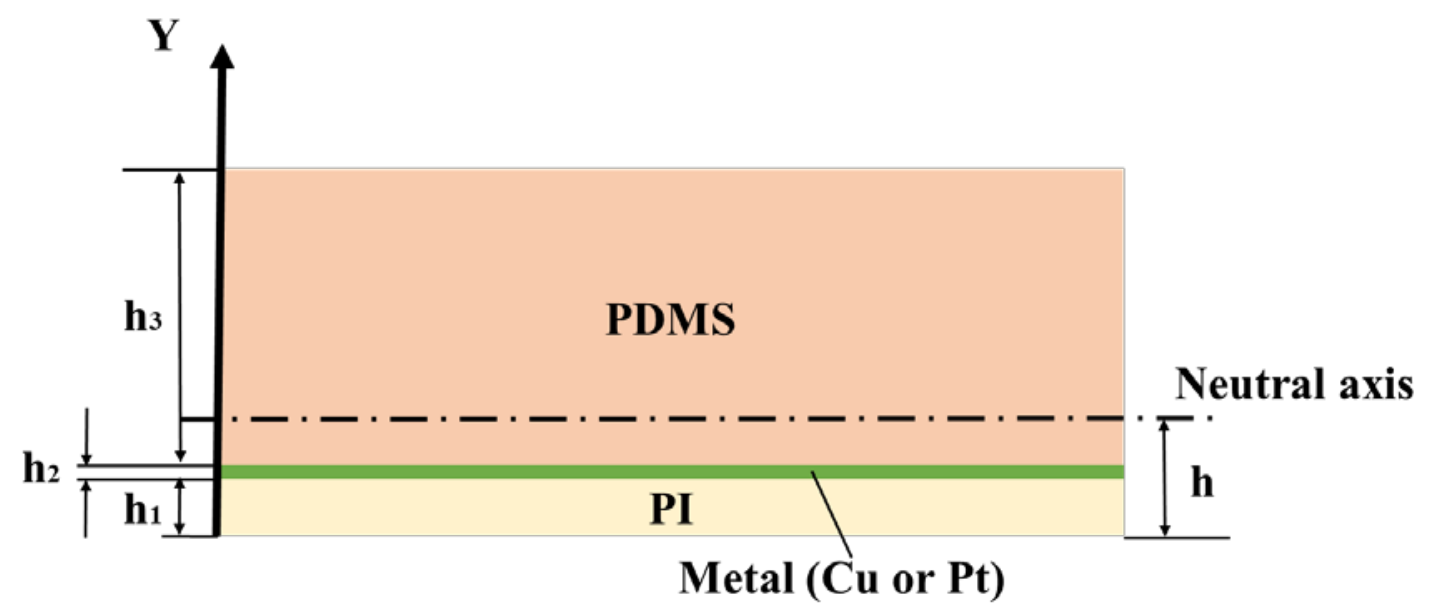

The interconnect had a trilayer design (PDMS/metal/PI), and the metal films were $\mathrm{Cu} / \mathrm{Ti}(\mathrm{Pt} / \mathrm{Cr}$ ). The thickness of $\mathrm{Ti}(\mathrm{Cr})$ was only $1 / 50$ of that of $\mathrm{Cu}(\mathrm{Pt})$; thus, the $\mathrm{Ti}(\mathrm{Cr})$ was negligible in the calculation. 